Original Research Paper

\title{
Electron Dimensions
}

\author{
${ }^{1}$ Relly Victoria Virgil Petrescu, ${ }^{2}$ Raffaella Aversa, ${ }^{3}$ Shuhui Li, \\ ${ }^{4}$ Ronald Bucinell, ${ }^{5}$ Samuel P. Kozaitis, \\ ${ }^{6}$ Taher M. Abu-Lebdeh, ${ }^{2}$ Antonio Apicella and ${ }^{1}$ Florian Ion Tiberiu Petrescu
}

${ }^{I}$ ARoTMM-IFToMM, Bucharest Polytechnic University, Bucharest, (CE), Romania

${ }^{2}$ Advanced Material Lab, Department of Architecture and Industrial Design, Second University of Naples, 81031 Aversa (CE), Italy

${ }^{3}$ University of Alabama, USA

${ }^{4}$ Union College, USA

${ }^{5}$ Florida Institute of Technology, USA

${ }^{6}$ North Carolina A and T State University, USA

Article history

Received: 28-05-2017

Revised: $30-05-2017$

Accepted: 05-06-2017

Corresponding Author: Florian Ion Tiberiu Petrescu ARoTMM-IFToMM,

Bucharest Polytechnic

University, Bucharest, (CE),

Romania

Email: scipub02@gmail.com

\begin{abstract}
In this study, the theoretical principles necessary to determine the exact magnitude of a moving electron, depending on the speed of movement, will be exposed. The equations are specifically discussed to determine the radius $R$ of the moving electron, which refers to the electron movement velocity $v$ and the resting mass $m_{0}$. The mechanical moment of inertia of a sphere around one of its diameters is determined by the relationship between the total kinetic energy of a moving electron as the sum of the two components (translatable and rotating). Using the theory of Louis de Broglie, which shows impulse preservation, the wavelength (associated with the particle) was calculated. The wave frequency (associated with the moving electron) was determined and the moving electron kinetic energy was estimated by decreasing the total resting energy of the electron from the total energy of the moving electrons.
\end{abstract}

Keywords: Electron Radius, Electron Speed, Rest Mass, Speed of Light, Planck's Constant, Electron Kinetic Energy, Lorentz Expression, Louis De Broglie Theory, Pulse Conservation, Wavelength Particle Associated, Wave Frequency

\section{Introduction}

The electron, one of the components of the atom with neutrons and protons, is an elementary particle with an elementary charge of negative sign. It is fundamental in chemistry because it participates in almost all types of chemical reactions and constitutes a primordial element of the bonds present in the molecules. In physics, the electron intervenes in a multitude of radiations and effects. Its properties, which are manifested on a microscopic scale, explain electrical conductivity, thermal conductivity, Vavilov-Cherenkov effect, incandescence, electromagnetic induction, luminescence, magnetism, electromagnetic radiation, optical reflection, the photovoltaic effect and the superconductivity, macroscopic phenomena widely exploited in the industrialized countries. Having the lowest mass of all charged particles, it regularly serves the study of matter.
The concept of an indivisible quantity of electric charge was elaborated from 1838 by the British naturalist Richard Laming in order to explain the chemical properties of the atoms. The electron is identified as the corpuscle envisioned by Joseph John Thomson and his team of British physicists in 1897, following their work on cathode rays.

It was at this time that Thomson proposed his atomic model. In 1905 Albert Einstein proposed an explanation of the photoelectric effect - electrons emitted by matter under the influence of light - which would serve as an argument in favor of quantum theory. In 1912, Niels Bohr explains the spectral lines by postulating the quantification of the electron orbit of the hydrogen atom, another argument supporting this theory. In 1914, the experiments of Ernest Rutherford and others solidly established the structure of the atom as a positively charged nucleus surrounded by weaker mass 
electrons. In 1923, the experimental results of Arthur Compton convince a majority of physicists of the validity of quantum theory. In 1924, Wolfgang Pauli defined Pauli's exclusion principle, which implies that the electrons possess a spin. In the same year, Louis de Broglie expressed the hypothesis, verified later, that the electrons present a wave-corpuscle duality. In 1928, Paul Dirac published his model of the electron which led him to predict the existence of the positron and then the antimatter. Other studies of the properties of the electron have led to more complete theories of matter and radiation.

The ancient Greeks have already remarked that amber attracts small objects when rubbed with fur; Apart from lightning, this phenomenon is the oldest experience of humanity in relation to electricity, a displacement of electrically charged particles.

In 1269, Pierre de Maricourt, a military engineer in the service of the French prince Charles I of Sicily, studied the properties of permanent magnets. "This study, which was transmitted to us in the form of a letter written to one of his colleagues, includes most of the elementary experiments now described in physics textbooks. In his treatise of 1600 De Magnete, the English physician William Gilbert creates the word lowlatin "electricus" to designate the property of attracting small objects after friction. The word "electric" derives from the English "electrick", which derives itself from the Latin "electricus": "peculiar to amber". The Latin word electrum derives from the Greek $\ddot{\eta} \lambda \varepsilon \kappa \tau \rho \circ \nu$ (électron) designating amber.

Francis Hauksbee in the 1700s and C. F. du Fay in 1737 independently discovered two kinds of electricity: One obtained by rubbing glass and the other generated by rubbing resin. Du Fay concludes that electricity can be reduced to two electric fluids, "glassy" and "resinous," which are separated by friction and which can be recombined together. A decade later, Benjamin Franklin argued that electricity does not differ from other types of electric fluids but that it is the same thing, under different pressures. It brings it modern terminology of positive or negative charge respectively.

Between 1838 and 1851, the British naturalist Richard Laming develops the idea that an atom is composed of a core of matter, surrounded by subatomic particles that have an electrical charge unit. Beginning in 1846, German physicist Wilhelm Eduard Weber argues that electricity is composed of positively and negatively charged fluids and that an inverse square law governs their interaction. After studying electrolysis in 1874, the Irish physicist George Stoney suggests that there is "a single defined quantity of electricity": The charge of a monovalent ion. This postulate gives him the ability to estimate the value of this elementary charge e from the laws of Faraday's electrolysis. However, Stoney believes that these charges are permanently attached to the atoms and therefore can't be removed from them. In 1881 the German physicist Hermann von Helmholtz was convinced that the positive and negative charges were composed of elementary parts, each of which was "behaving like electricity atoms."

In 1894, Stoney invented the term "electron" to designate these elementary charges, writing ".. an estimate was made of the real value of this very remarkable fundamental unit of electricity, for which I ventured to propose the name of "electron". The word electron is a combination of the word "electric" and the suffix "-on", the latter being subsequently used to designate a subatomic particle, such as the proton or the neutron.

During the nineteenth century German physicists Julius Plücker and Johann Wilhelm Hittorf studied the electrical conductivity of gases in sealed glass ampoules equipped with a cathode and an anode, which made it possible to subject the gas to an electric current. In 1869 , Hittorf observed the emission, by the cathode, of charged "beams of particles" if the bulb contained a gas at low pressure. In 1876, the German physicist Eugen Goldstein shows that the rays of this glow cause a shadow and he calls them cathode rays. During the 1870s, the English chemist and physicist William Crookes developed the first cathode ray tube with a deep vacuum inside - later named "Crookes tube." Then it shows that the light rays appearing in the tube transmit energy and move from the cathode to the anode. Moreover, by applying a magnetic field, it is capable of deflecting the rays, thus showing that the beam behaves as if it were charged. In 1879 he proposed to explain these properties by what he called "radiant material". He considers this to be a fourth state of matter, consisting of negatively charged molecules, projected at high velocity of the cathode.

The German-born British physicist Arthur Schuster develops Crookes experiments by placing metal plates parallel to the cathode rays, by which he can apply different electrical potentials. The electric field deflects the rays towards the positively charged plate, which reinforces the proof that the rays carry a negative charge. By measuring the deflection according to the potential difference, Schuster is able in 1890 to measure the massto-charge ratio of the components of the spokes. However, its calculation gives a value more than a thousand times less than the expected value, so that contemporaries give little confidence to its calculation. In 1895, the PhD student and future French physicist Jean Perrin experimentally established the corpuscular nature of the electron, while several scientists of that time considered the electron as a wave.

In 1896-1897 the British physicist Joseph John Thomson and his colleagues John Townsend and Harold A. Wilson carried out experiments indicating that cathode rays are actually individualized particles, rather 
than waves, atoms or molecules as the specialists believe At the time. Thomson makes good estimates of both the charge e and the mass $\mathrm{m}$, finding that the particles of the cathode rays, which he calls "corpuscles," have about one thousandth of the mass of the lightest ion known then: Hydrogen. It shows that the charge to mass e/m ratio is independent of the material of the cathode. It also shows that the negatively charged particles produced by radioactive materials, heated materials and illuminated materials are the same. His considerable work on the deflection of cathode rays in an electric field is probably the reason why he is attributed the discovery of the electron. The name of electron is proposed again by the Irish physicist George F. Fitzgerald, this time with success. Historically, the electron is the first elementary particle highlighted.

In 1900, Paul Drude proposed to consider the whole of the electrons of a metal as a perfect gas. He then succeeds in theoretically justifying an experimental conclusion that good electrical conductors are also good thermal conductors. Even if his hypothesis is false according to current knowledge, this concept of "perfect gas of electrons" is still used in quantum mechanics.

By studying naturally fluorescent minerals, the French physicist Henri Becquerel discovers that these emit radiation in the absence of any external energy source. These radioactive materials provoke the enthusiasm of scientists, including that of the New Zealand physicist Ernest Rutherford, who discovers that they emit particles. It gives them the name alpha, beta and gamma particles, according to their power of penetration of matter. In 1900, Becquerel shows that the beta rays emitted by radium are deflected by an electric field and that their mass-to-charge ratio is the same as that of cathode rays. This result supports the idea that electrons exist as components of atoms.

The electron charge is measured more precisely by the American physicist Robert Millikan by his experiment on the oil drop of 1909, of which he published the results in 1911. This experiment uses an electric field to compensate for gravity and prevent thus a drop of oil loaded with falling. This system allows the electrical charge to be measured from a few ions up to 150 , with a margin of error of less than $0.3 \%$. Comparable experiments were done earlier by the Thomson group, using electrolytically charged water droplets and in 1911 by Abram Ioffé, which achieved independently the same result as Millikan using metal microparticles and Published its results in 1913. However, less volatile oil drops are better suited for long-term experiments.

Towards the beginning of the twentieth century, physicists discovered that, under certain conditions, a rapid particle provoked on its trajectory the condensation of supersaturated water vapor. In 1911, the Scottish physicist Charles Thomson Rees Wilson, one of Thomson's collaborators, used this effect to develop his fog chamber, which photographed traces of charged particles, such as fast electrons, their study.

The work of the New Zealand physicist Ernest Rutherford from 1909 to 1912 led him to conclude that the atom consists of a small nucleus comprising all the positive charge and almost all the mass of the atom, a nucleus which is surrounded Of an electronic cloud (see Rutherford's Experiment). The British physicist Henry Moseley, who works in the Rutherford laboratory in 1913 , defines with certainty the order of the chemical elements in the periodic table.

In 1913, the Danish physicist Niels Bohr postulated that electrons are in quantified states, whose energy is determined by the angular momentum around the nucleus. The electrons can pass from one state to another, by emission or absorption of photons at specific frequencies. By means of these quantified orbits, he explains with all precision the spectral lines of the hydrogen atom.

The work of the German physicists James Franck and Gustav Hertz, from 1912 to 1914, proves the quantification of the energy levels of the electrons in the atoms and thus confirm the hypotheses of the Bohr atom model (see Franck and Hertz's experiment). All these experiments solidly establish the structure of the atom as a positively charged nucleus surrounded by electrons of lower mass.

However, the Bohr model can't account for the relative intensities of the spectral lines, nor explain the more complex spectra of atoms. Despite these weaknesses, this atomic model will serve as an argument in favor of quantum theory.

The chemical bonds between atoms are explained by Gilbert Lewis, who proposes in 1916 that the covalent bond between atoms is a pair of shared electrons. Later, in 1927, Walter Heitler and Fritz London completely explain the formation of electron pairs and chemical bonds in terms of quantum mechanics. In 1919, the American chemist Irving Langmuir refined the static model of Lewis atom and suggested that all electrons were distributed on "concentric (nearly) spherical layers all of the same thickness". The layers are in turn divided into a number of cells, each containing a pair of electrons. With this model, Langmuir explains qualitatively the chemical properties of all the elements of the periodic table, which scientists compare according to the law of similarity.

In 1924, the Austrian physicist Wolfgang Pauli noticed that the layered structure of the atom is explained by the set of four parameters which define all the energetic states, as long as each state is occupied by only one electron. This prohibition of two electrons occupying the same state became known as the "principle of Pauli's exclusion". The mechanism 
determining the fourth parameter and its two values is provided by the Dutch physicists Samuel Goudsmit and George Uhlenbeck when they suggest that the electron, in addition to the angular momentum of its orbit, could have an intrinsic angular momentum. This property becomes known as "spin": It explains the splitting of spectral lines observed with a high-resolution spectrograph, which has remained mysterious until then; this phenomenon is known as the hyperfine structure of the lines. The principle of Pauli explains why the socalled ordinary matter creates what is called the "volume" of matter.

The electrons possess, like all matter, the quantum property of being both waves and corpuscles, so that they can have collisions with other particles and be diffracted like light. This duality is easy to detect with the electrons because of their low mass. An electron, because of its spin, is a fermion and thus satisfies the principle of exclusion of Pauli.

In 1887, the photoelectric effect is observed by Heinrich Hertz as he studies electromagnetic waves and several scientists have tried to explain the mechanisms, without result. Twenty years later, in 1905, Albert Einstein proposed a first explanation, which earned him the Nobel Prize in Physics of 1921. According to him, electrons are emitted by matter only if the frequency of light is greater than a certain threshold. To achieve this, he introduces the concept of photon, using that of energy quantum recently proposed in a completely different context by Max Planck. Einstein's explanation will be one of the first arguments in favor of quantum theory. In 1923, Arthur Compton observes the elongation of the wavelength of the photon caused by the diffusion that bears his name, which is caused by the interaction of photons and electrons. "These experimental results are the first to convince most physicists of the validity of quantum theory."

In his publication recherches sur le theorie des quanta, in 1924, the French physicist Louis de Broglie hypothesizes that all matter has a De Broglie wave similar to light. That is to say, depending on the conditions, the electrons and other material particles show the properties either of particles or of waves. The corpuscular properties of a particle are patent when it appears at any time localized at a point in space along a trajectory. The wave nature is observed, for example, when a beam passes through parallel slits and creates interference patterns. In 1927, the interference effect with an electron beam was shown by the English physicist George Paget Thomson, using a thin metallic film and by American physicists Clinton Davisson and Lester Germer using a nickel crystal.

The success of De Broglie's prediction led to the publication by Erwin Schrödinger in 1926 of the Schrödinger equation which successfully describes the propagation of electrons as a wave. Rather than providing a solution giving the position of an electron, this wave equation can be used to calculate the probability of finding an electron in a certain volume. This approach is subsequently called quantum mechanics and gives a very good approximation of the states of energy in the hydrogen atom. Once the spin and the interactions between the electrons are taken into account, quantum mechanics successfully models the behavior of the electrons in the more complex atoms than that of hydrogen.

In 1928, the British physicist Paul Dirac, working on the work of Wolfgang Pauli, designed an electron model - the Dirac equation-compatible with the theory of relativity and quantum mechanics. To solve certain shortcomings in his relativistic equation, Dirac developed in 1930 a model of vacuum with an infinite sea of particles of negative energy, sometimes called "sea of Dirac". This leads him to predict the existence of the positron, the equivalent of the electron in antimatter, a physical "entity" also predicted by Dirac. The positron is discovered by Carl D. Anderson, who proposes to call the standard electrons "negatrons" and to use the term "electron" as a generic term to designate the two charges without distinction. This use of the term "negatron" is still encountered from time to time and may be abbreviated as "negatron".

In 1947, the American physicist Willis Lamb, in collaboration with the $\mathrm{PhD}$ student Robert Retherford, discovered that some quantum states of the hydrogen atom, which should have the same energy, are distinguished by a certain discrepancy. Lamb at about the same time, the German-American physicist Polykarp Kusch, working with Henry M. Foley, discovers that the magnetic moment of the electron is somewhat greater than that predicted by Dirac's theory. This gap will later be called the "anomalous magnetic moment" of the electron. To solve these problems, a more elaborate theory, called "quantum electrodynamics", was developed by Sin-Itiro Tomonaga, Julian Schwinger and Richard Feynman in the late 1940s.

The electron has a wave-particle duality, which can be demonstrated by the experiment of Young's slits.

This property allows it to pass through two parallel slots simultaneously, rather than just a single slit, as would be the case for a conventional particle. In quantum mechanics, the wave property of a particle can be described mathematically as a function with complex values, the wave function, commonly denoted by the Greek letter psi $(\psi)$. When the absolute value of this function is squared, this gives the probability of observing a particle in a small volume near the chosen position - a probability density. The electron can cross a potential barrier by tunnel effect, a phenomenon that classical mechanics can't explain and which quantum mechanics explains by using the notion of wave function. 
Electrons are indistinguishable particles, because they can't be distinguished from one another by their intrinsic physical properties. In quantum mechanics, this means that a pair of electrons present must be able to invert their position without causing any observable change in the state of the system.

In the case of antisymmetry, solutions of the wave equation for interacting electrons result in a zero probability that two electrons occupy the same position, or, taking into account the spin, the same state.

This is the cause of Pauli's exclusion principle, which prevents two electrons from occupying the same quantum state. This principle explains many properties of electrons. For example, it allows us to assert that clouds of electrons linked to the same nucleus occupy all different orbitals, rather than all of them being concentrated in the least energy orbital.

An electron can be "bound" to the nucleus of an atom by the attractive Coulomb force. A system of electrons linked to a nucleus in number equal to the positive charge of the latter is called a "neutral atom". If the number of electrons is different, the system is called an "ion". The nucleus of atoms comprises protons and, in general, neutrons. The atoms are thus formed of three particles: Electrons, neutrons and protons. The wave behavior of a bonded electron is described by a function called "atomic orbital". Each orbital has its own set of quantum numbers, such as energy, angular momentum and projection of the latter on a given axis. According to the exclusion principle of Pauli, each orbital can be occupied at most by only two electrons, of different spins.

Electrons can change orbital by emission or absorption of a photon whose energy equals the potential energy difference between these atomic orbitals. Other methods of orbital transfer include collisions with particles such as electrons and the Auger effect. To escape from an atom, the energy of the electron must be hoisted above its binding energy to the atom. This can happen in the photoelectric effect when an incident photon has an energy that exceeds the ionization energy of the electron that absorbs it.

The orbital angular moment of the electrons is quantified. As the electron is charged, it produces an orbital magnetic moment proportional to its angular momentum.

The total magnetic moment of an atom is equal to the sum of the magnetic and orbital moments of all the electrons and of the nucleus. That of the nucleus, however, is negligible compared to that of the electrons. The magnetic moments of the electrons that occupy the same orbital (electrons in pairs) cancel each other out.

In physics, the chemical bond between atoms results from electromagnetic interactions, described by the laws of quantum mechanics. Depending on their proximity to the nucleus, chemists consider heart electrons and valence electrons; it is the latter which intervene in the chemical bond.

The strongest are the covalent bonds and the ionic bonds, which allow the formation of molecules. In a molecule, electrons move under the influence of several nuclei and occupy molecular orbitals in the same way that they occupy orbitals in isolated atoms. A fundamental factor in these molecular structures is the existence of electron pairs: These are opposite spin electrons, allowing them to occupy the same molecular orbital without violating Pauli's exclusion principle (from same way as in atoms). Different molecular orbitals have spatial distributions of different electron densities. For example, in the bonding pairs - which bind the atoms together, one finds electrons with a maximum density in a relatively small volume between the atoms. On the contrary, for the non-binding pairs, the electrons are distributed in a large volume around the nuclei. It is the existence of binding pairs, where peripheral electrons are pooled by two neighboring atoms, which characterizes the covalent bond. Ion bonding occurs when two ions are strongly bonded by electrostatic attraction, which occurs if one electron of one atom has a molecular orbital mostly located near the other atom.

If a body has too many electrons, or not enough, to balance the positive charges of the nuclei, it has a nonzero electric charge: Negative if there are too many electrons; Otherwise. If the charges are balanced, the body is said to be neutral.

Electrons moving independently, as in vacuum, are called "free". The valence electrons in metals also behave as if they were free. In addition, there may be in a solid hole, which are places where an electron is missing. These holes can be filled by neighboring electrons, but this will only move the holes. One can have in solids a predominance of the conduction of the electricity by the displacement of holes, rather than by the displacement of electrons. In fact, the chargebearing particles in metals and other solids are "quasiparticles", of negative or positive electric charge, similar to real electrons.

When free electrons move - whether in a vacuum or in a metal they produce a net charge current, which is called an electric current, which generates a magnetic field. Similarly, a current can be generated by an electric field, possibly caused by a variable magnetic field (electromagnetic induction). These interactions are described mathematically by Maxwell's equations.

At a given temperature, each material has an electrical conductivity which determines the value of the electric current when an electrical potential is applied. Examples of good conductors include metals such as copper and gold, while glass and Teflon are bad conductors (these are insulators). In any dielectric material, the electrons remain bound to their 
respective atoms and the material behaves as an electrical insulator.

Most semiconductors have varying degrees of conductivity between conductor and insulator extremes. Moreover, the metals have a structure in electronic bands, some of which are only partly filled. The presence of this type of bands allows the electrons to behave as if they were free or delocalized. When an electric field is applied, they can move like molecules of a gas (called "Fermi gas") through matter, much like free electrons.

These phenomena are the basis of all electricity: Electrokinetics, electronics and radioelectricity.

Due to collisions between electrons and atoms, the drift velocity of electrons in a conductor is of the order of $\mathrm{mm} / \mathrm{s}$. However, the speed at which a current change at one point of the material affects the currents at other points, velocity is typically $75 \%$ of the velocity of light in a vacuum. This occurs because the electrical signals propagate like a wave, with a velocity that depends only on the dielectric constant, or permittivity, of the medium.

Metals are relatively good conductors of heat, above all because delocalized electrons can carry thermal energy from one atom to another. However, unlike electrical conductivity, the thermal conductivity of a metal is practically independent of temperature.

This is expressed mathematically by the law of Wiedemann and Franz, who says that the ratio of the thermal conductivity to the electrical conductivity is proportional to the temperature. Since the thermal disorder of the lattice of the metal increases the resistivity of the medium, this leads the electric current to depend on the temperature.

When cooled below a critical temperature, the substances can undergo a phase transition which causes them to lose any resistivity to the electric current, a phenomenon called "superconductivity". In the BCS theory, this behavior is explained by pairs of electrons (forming bosons) which enter the state known as "BoseEinstein condensate". These Cooper pairs see their movement coupled to the surrounding matter by network vibrations called "phonons", thus avoiding collisions with the atoms responsible for electrical resistance.

In solid conductors, electrons are quasi-particles. When they are strongly confined to temperatures close to absolute zero, they behave as if they decompose into two other quasi-particles, spinons and chargeons. The first carries the spin and the magnetic moment. The second, the electric charge: It is the spin-charge separation.

The incandescence, which appears in the heated materials, is caused by the orbital changes of the electrons in the atom. Luminescence is an emission of light that occurs at relatively low temperatures and is also a consequence of changes in orbital electrons in the atom.

When an electron is accelerated, it can radiate energy in the form of photons. This electromagnetic radiation is manifested in the form of radio waves, microwaves, infrared, visible light (perceived by the human eye), ultraviolet, X-rays or gamma rays.

Optical diffusion, an interaction between light and electrons, explains optical reflection. Rayleigh scattering helps explain the color of the sky and the color of the feathers of certain birds.

The refraction of electromagnetic waves is also a result of the interaction of light and electrons.

These optical phenomena are also caused by the interaction of photons with other charged particles such as the proton. Most photon interactions with matter are reduced to three phenomena: Photoelectric effect, Compton scattering and electron-positron (or materialization) pair production. They occur mostly in the presence of electrons because they are the least heavy charged particles. The photovoltaic effect is obtained by absorption of the photons in a semiconductor material which then generates electron-hole pairs (excitation of an electron from the valence band towards the conduction band) creating an electric voltage or current. It is particularly used in photovoltaic solar panels.

As far as we know, base matter consists of quarks and leptons.

Quarks combine to form hadrons, mainly baryons and mesons, with strong force and are supposed to be still well isolated.

Among the baryons are the proton (positive electric charge) and the neutrons (with zero electrical charge) that they combine to form the atomic nuclei of all the chemical elements of the periodic table. Normally, an electron core (the negative electrical charge and the opposite of the proton) surrounds these nuclei. The cluster consisting of a nucleus and a cloud, containing negative electrons and positive protons, is an atom. Atoms can be arranged together to form larger and more complex structures, such as molecules.

Chemistry is a science that describes how nuclei and electrons are combined to form different elements and molecules (Aversa et al., 2016a; 2016b; 2016c; 2016d; 2016e; Petrescu et al., 2016a; 2016b).

In a more cosmological view, matter and antimatter are taken into account. Each sub-particle of an atom can be counterbalanced by a pair (anti-) antimatter (e.g., electron-positron). An antimatter particle differs from its partner by the fact that its different "fillings" (electric charge, rotation, color loading, etc.) are opposite. However, these particles have the same mass.

Although the fundamental laws of physics do not indicate a preference for matter over antimatter, cosmological observations show that the universe consists almost entirely of matter.

The material can be found in several states or phases. The four most well-known states are solid, liquid, gas and plasma. There are other less exotic states, such as 
liquid crystal, Bose-Einstein, superfluid and supercritical fluid (Bose-Einstein, Wikipedia). When the material goes from state to state, it performs a phase transition.

This phenomenon (which is associated with changes in physical parameters: Pressure, temperature, volume, density, energy, etc.) is studied in thermodynamics by phase diagrams.

When more particles combine to form atoms, the total mass (at rest) of the assembly is less than the sum of the mass (at rest) because in fact a part of the weight of the components converts the binding energy required to provide the Coefficient of Cluster This phenomenon is reported as "massive defect"). By speculating this defect, nuclear fusion energy is extracted.

The electron, one of the particles of the atom having an elementary charge of the negative sign, is fundamental in chemistry because it participates in almost all types of chemical reactions and is a key element of the chemical bond of matter.

In physics, on the other hand, the electron is involved in a multitude of phenomena related to the effects of radiation. Electromagnetic induction, luminescence, magnetism, electromagnetic radiation, optical reflection and large-scale macroscopic super conduction exploited in industrial applications (Cherenkov, Wikipedia) explain the electrical characteristics exposed at the atomic level.

In addition, the electron with the smallest mass in comparison to any other charged particles is regularly used in the study of matter.

The concept of an indivisible amount of electric charge was developed in 1838 by British naturalist Richard Laming to explain the chemical properties of atoms (Laming, Wikipedia).

The electron was described as the corpusculus predicted by Joseph John Thomson and his team of British physicists in 1897, after working on cathode ray and developing his atomic model (Thomson, 1897).

Albert Einstein proposed in 1905 an explanation of the photoelectric effect-electrons emitted by matter under the influence of light - which was reinterpreted as a basis in the development of quantum theory (Einstein, Wikipedia). In 1912, Niels Bohr explains the spectral lines that postulate the quantification of electrons in hydrogen orbit, another argument that supports this theory (Bohr, Wikipedia).

Experiments conducted by Ernest Rutherford and others in 1921 have firmly established the structure of the atom as a positively charged nucleus surrounded by small electrons (Rutherford, Wikipedia). In 1923, experimental results Arthur Compton convinced most physicists about the validity of quantum theory (Compton, Wikipedia). In 1924, Wolfgang Pauli's principle defines Pauli exclusion, which means that electrons possess spin (Pauli, Wikipedia).
In the same year, Louis de Broglie initially postulated and then later verified that electrons have duality with particle waves (Louis de Broglie, Wikipedia). In 1928, Paul Dirac published his electron model that introduced the concept of positron and antimatter (Dirac, Wikipedia). Other studies of electronic properties have led to more complete theories of matter and radiation.

\section{Materials and Methods}

When it comes to determining the size of subatomic atomic particles, static diameters (or more precisely, particle diameters in the resting state) are commonly used. They were determined by a variety of more or less empirical methods and very close values (Halliday and Robert, 1966). These dimensions (which, depending on the particle, may be in the order of Nano, Pico or less) are very difficult to determine both experimentally and theoretically even today when we have many sophisticated characterization tools (atomic force microscopes, Nanoindenter etc.). However, the real problem of characterization occurs when these particles are in dynamic motion. In this dynamic condition, it is imperative to determine real dimensions in motion, making things even more complex than they are already.

The previous experiences of the authors have come to the theoretical identification of the dimensions of the elementary particles in motion, as long as they do not attain the speed of light (Petrescu and Calautit, 2016a, Petrescu et al., 2016a; 2016b).

For photons moving at the speed of light, a special method for determining their dimensions was presented, depending on the frequency of the specific electromagnetic wave (Petrescu and Calautit, 2016b).

This paper presents the theoretical principles necessary to determine the exact magnitude of an electron in motion relative to the specific displacement velocity.

Relationship 1 can accurately determine the radius of the moving electron $R$, depending on the velocity $v$ and the rest mass $m_{0}$. The speed of light in a vacuum is expressed with $c$ and Planck's constant with $h$ (Planck, Wikipedia):

$R=\sqrt{\frac{10}{8}} \cdot \frac{h \cdot \sqrt{c^{2}-v^{2}} \cdot \sqrt{c^{2}-\frac{v^{2}}{2}-c \cdot \sqrt{c^{2}-v^{2}}}}{\pi \cdot m_{0} \cdot c^{2} \cdot v}$

Mechanical moment of inertia of a sphere around of one of its axes could be determined by using the relationship 2 (Fig. 1), (Petrescu, 2012; Mirsayar et al., 2017):

$J=\frac{2}{5} m \cdot R^{2}$ 


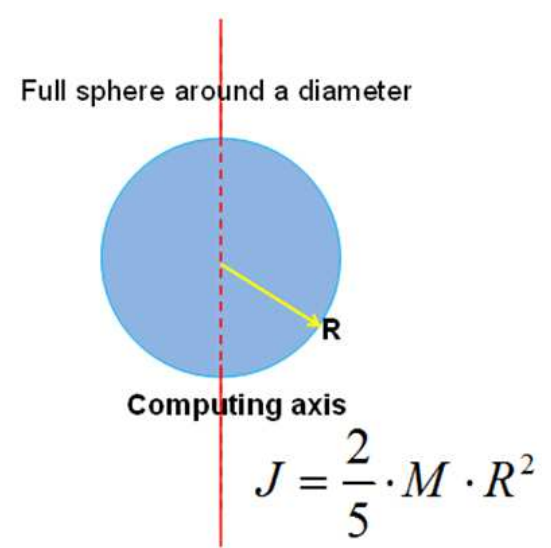

Fig. 1. Mass moment of inertia to a full sphere, determined around its symmetry axis

The mass of an electron in motion can be determined then according to its speed and its rest mass using rehandled Lorentz expression 3 (Lorentz, Wikipedia):

$$
m=\frac{m_{0} \cdot c}{\sqrt{c^{2}-v^{2}}}
$$

The electron (like any elemental mobile particle) has two types of motion, one translated and one that rotates around its own axis. These two movements are consistent with the two kinetic energies (the energy due to the movement of the translation electron on its trajectory and the energy due to the rotation of the electron around the axis of the diameter); expression 4 shows the total kinetic energy of a moving electron as a sum of the two components (translational and rotational):

$$
E_{c}=\frac{1}{2} m \cdot v^{2}+\frac{1}{2} J \cdot \omega^{2}
$$

Using the two expressions 2 and 3, the relationship 4 takes the below forms 5 :

$$
\left\{\begin{array}{l}
E_{c}=\frac{1}{2} m \cdot v^{2}+\frac{1}{2} \cdot \frac{2}{5} \cdot m \cdot R^{2} \cdot \omega^{2} \\
E_{c}=\frac{1}{2} \frac{m_{0} \cdot c \cdot v^{2}}{\sqrt{c^{2}-v^{2}}}+\frac{1}{2} \cdot \frac{2}{5} \cdot \frac{m_{0} \cdot c \cdot R^{2}}{\sqrt{c^{2}-v^{2}}} \cdot \omega^{2}
\end{array}\right.
$$

One uses the expression 6 to write the particle pulse:

$$
p=m \cdot v=\frac{m_{0} \cdot c \cdot v}{\sqrt{c^{2}-v^{2}}}
$$

Using the theory of Louis de Broglie (de Broglie, Wikipedia), which describes the conservation of the pulse the wavelength particle associated may be calculated using relationship 7:

$$
\lambda=\frac{h}{p}=\frac{h \cdot \sqrt{c^{2}-v^{2}}}{m_{0} \cdot c \cdot v}
$$

Wave frequency (associated with the electron in motion) can be then determined using the relationship 8 (Halliday and Robert, 1966):

$$
\gamma=\frac{c}{\lambda}=\frac{c \cdot m_{0} \cdot c \cdot v}{h \cdot \sqrt{c^{2}-v^{2}}}=\frac{m_{0} \cdot c^{2} \cdot v}{h \cdot \sqrt{c^{2}-v^{2}}}
$$

The angular electron speed and its square are calculated using the expressions 9:

$$
\left\{\begin{array}{l}
\omega=2 \pi \gamma=\frac{2 \pi \cdot m_{0} \cdot c^{2} \cdot v}{h \cdot \sqrt{c^{2}-v^{2}}} \\
\omega^{2}=\frac{4 \pi^{2} \cdot m_{0}^{2} \cdot c^{4} \cdot v^{2}}{h^{2} \cdot\left(c^{2}-v^{2}\right)}
\end{array}\right.
$$

Considering expressions 9, expressions 5 may be written in the following expressions 10 :

$$
\left\{\begin{array}{l}
E_{c}=\frac{1}{2} \frac{m_{0} \cdot c \cdot v^{2}}{\sqrt{c^{2}-v^{2}}} \\
+\frac{1}{2} \cdot \frac{2}{5} \cdot \frac{m_{0} \cdot c \cdot R^{2}}{\sqrt{c^{2}-v^{2}}} \cdot \frac{4 \pi^{2} \cdot m_{0}^{2} \cdot c^{4} \cdot v^{2}}{h^{2} \cdot\left(c^{2}-v^{2}\right)} \\
E_{c}=\frac{1}{2} \frac{m_{0} \cdot c \cdot v^{2}}{\sqrt{c^{2}-v^{2}}} \cdot\left[1+\frac{8}{5} R^{2} \cdot \pi^{2} \frac{m_{0}^{2} \cdot c^{4}}{h^{2} \cdot\left(c^{2}-v^{2}\right)}\right]
\end{array}\right.
$$

Moving electron kinetic energy may be calculated through the expression 11 by subtracting the total electron rest energy from total electron energy in movement:

$$
E_{c}=E-E_{0}=m \cdot c^{2}-m_{0} \cdot c^{2}=m_{0} \cdot c^{2} \cdot \frac{c-\sqrt{c^{2}-v^{2}}}{\sqrt{c^{2}-v^{2}}}
$$

By considering the two expressions 10 and 11 and combining them, the first term of relationships 12 is obtained, which represent the expression to evaluate the radius of an electron in movement. The second term from in Equation 12 is the initially mentioned relation 1:

$$
\left\{\begin{array}{l}
\frac{1}{2} \frac{m_{0} \cdot c \cdot v^{2}}{\sqrt{c^{2}-v^{2}}} \cdot\left[1+\frac{8}{5} R^{2} \cdot \pi^{2} \frac{m_{0}^{2} \cdot c^{4}}{h^{2} \cdot\left(c^{2}-v^{2}\right)}\right] \\
=m_{0} \cdot c^{2} \cdot \frac{c-\sqrt{c^{2}-v^{2}}}{\sqrt{c^{2}-v^{2}}} \\
R=\sqrt{\frac{10}{8} \cdot \frac{h}{\pi \cdot m_{0} \cdot c^{2}} \cdot \frac{\sqrt{c^{2}-v^{2}} \cdot \sqrt{c^{2}-\frac{v^{2}}{2}-c \cdot \sqrt{c^{2}-v^{2}}}}{v}} \\
R=\sqrt{\frac{10}{8}} \cdot \frac{h \cdot \sqrt{c^{2}-v^{2}} \cdot \sqrt{c^{2}-\frac{v^{2}}{2}-c \cdot \sqrt{c^{2}-v^{2}}}}{\pi \cdot m_{0} \cdot c^{2} \cdot v}
\end{array}\right.
$$




\section{Results}

By using Equation 1, the radius of any elementary moving particle can now be calculated. Table 1 shows the radius of a moving electron (calculated with Equation 1) according to $\beta$ ( $\beta$ being the ratio between $v$ and $c$, expression 13):

$$
\beta=\frac{v}{c}
$$

Using the original method proposed by the authors, the moving electron beam can be determined with great precision, depending on the speed at which it moves. It can be seen from the results presented in Table 1 that the electron has no constant radius. The electronic phase depends primarily on the speed of movement and, secondly, on the rest mass.

From the table shown, the average radius of an electron $1.09756 \mathrm{E}-13[\mathrm{~m}]$ and a maximum electronic value of $1.83152 \mathrm{E}-13[\mathrm{~m}]$ corresponding to a $\beta=0.8$ can be determined.

The minimum radius value (in real cases) is about 8.15E-17 [m], but may decrease more when the limits are reached.

Electrons that normally move at low speeds of about $0.01 \mathrm{c}$ will have a range of 3.05E-15 [m]. Only this value can be found using classical relationships already known.

\section{Discussion}

In this study, the theoretical principles necessary to determine the exact magnitude of a moving electron, depending on the speed of movement, will be exposed.

The equations are specifically discussed to determine the radius $R$ of the moving electron, which refers to the electron movement velocity $v$ and the resting mass $m_{0}$.

The mechanical moment of inertia of a sphere around one of its diameters is determined by the relationship between the total kinetic energy of a moving electron as the sum of the two components (translatable and rotating).

Table 1. The electron radius in function of $\beta$

\begin{tabular}{llll}
\hline$\beta$ & 0.000009 & 0.00002 & 0.0001 \\
$\mathrm{R}[\mathrm{m}]$ & $4.93 \mathrm{E}-16$ & $4.07 \mathrm{E}-16$ & $8.15 \mathrm{E}-17$ \\
$\beta$ & 0.001 & 0.01 & 0.1 \\
$\mathrm{R}[\mathrm{m}]$ & $3.05 \mathrm{E}-16$ & $3.05 \mathrm{E}-15$ & $3.04 \mathrm{E}-14$ \\
$\beta$ & 0.2 & 0.3 & 0.4 \\
$\mathrm{R}[\mathrm{m}]$ & $6.04 \mathrm{E}-14$ & $8.94 \mathrm{E}-14$ & $1.16 \mathrm{E}-13$ \\
$\beta$ & 0.5 & 0.6 & 0.7 \\
$\mathrm{R}[\mathrm{m}]$ & $1.41 \mathrm{E}-13$ & $1.62 \mathrm{E}-13$ & $1.78 \mathrm{E}-13$ \\
$\beta$ & 0.8 & 0.9 & 0.99 \\
$\mathrm{R}[\mathrm{m}]$ & $1.83 \mathrm{E}-13$ & $1.66 \mathrm{E}-13$ & $7.47 \mathrm{E}-14$ \\
$\beta$ & 0.999 & 0.9999 & 0.99999 \\
$\mathrm{R}[\mathrm{m}]$ & $2.61 \mathrm{E}-14$ & $8.51 \mathrm{E}-15$ & $2.71 \mathrm{E}-15$ \\
$\beta$ & 0.999999 & 0.9999999 & 1 \\
$\mathrm{R}[\mathrm{m}]$ & $8.62 \mathrm{E}-16$ & $2.72 \mathrm{E}-16$ & $8.63 \mathrm{E}-17$ \\
\hline
\end{tabular}

Using the theory of Louis de Broglie, which shows impulse preservation, the wavelength (associated with the particle) was calculated.

The wave frequency (associated with the moving electron) was determined and the moving electron kinetic energy was estimated by decreasing the total resting energy of the electron from the total energy of the moving electrons.

As far as we know, base matter consists of quarks and leptons. Quarks combine to form hadrons, mainly baryons and mesons, with strong force and are supposed to be still well isolated. Among the baryons are the proton (positive electric charge) and the neutrons (with zero electrical charge) that they combine to form the atomic nuclei of all the chemical elements of the periodic table. Normally, an electron core (the negative electrical charge and the opposite of the proton) surrounds these nuclei. The cluster consisting of a nucleus and a cloud, containing negative electrons and positive protons, is an atom. Atoms can be arranged together to form larger and more complex structures, such as molecules.

Chemistry is a science that describes how nuclei and electrons are combined to form different elements and molecules (Aversa et al., 2016a; 2016b; 2016c; 2016d; 2016e).

In a more cosmological view, matter and antimatter are taken into account. Each sub-particle of an atom can be counterbalanced by a pair (anti-) antimatter (e.g., electron-positron). An antimatter particle differs from its partner by the fact that its different "fillings" (electric charge, rotation, color loading, etc.) are opposite. However, these particles have the same mass.

Although the fundamental laws of physics do not indicate a preference for matter over antimatter, cosmological observations show that the universe consists almost entirely of matter.

The material can be found in several states or phases. The four most well-known states are solid, liquid, gas and plasma. There are other less exotic states, such as liquid crystal, Bose-Einstein, superfluid and supercritical fluid (Bose-Einstein, Wikipedia). When the material goes from state to state, it performs a phase transition. This phenomenon (which is associated with changes in physical parameters: Pressure, temperature, volume, density, energy, etc.) is studied in thermodynamics by phase diagrams.

When more particles combine to form atoms, the total mass (at rest) of the assembly is less than the sum of the mass (at rest) because in fact a part of the weight of the components converts the binding energy required to provide the Coefficient of Cluster This phenomenon is reported as "massive defect"). By speculating this defect, nuclear fusion energy is extracted. 
The electron, one of the particles of the atom having an elementary charge of the negative sign, is fundamental in chemistry because it participates in almost all types of chemical reactions and is a key element of the chemical bond of matter. In physics, on the other hand, the electron is involved in a multitude of phenomena related to the effects of radiation. Electromagnetic induction, luminescence, magnetism, electromagnetic radiation, optical reflection and largescale macroscopic superconduction exploited in industrial applications (Cherenkov, Wikipedia) explain the electrical characteristics exposed at the atomic level.

In addition, the electron with the smallest mass in comparison to any other charged particles is regularly used in the study of matter.

\section{Applications}

An electron microscope (ME) is a type of microscope that uses a beam of electron particles to illuminate a sample and create a much enlarged image. Electronic microscopes have a higher resolution power than optical microscopes that use electromagnetic radiation. They can achieve much higher magnifications up to 5 million times, while the best optical microscopes are limited to a magnification of 2000 times. These two types of microscopes have a limiting resolution, imposed by the wavelength of the radiation they use. The higher resolution and magnification of the electron microscope are due to the fact that the wavelength of an electron (Broglie wavelength) is much smaller than that of a visible light photon.

The electron microscope uses electrostatic and electromagnetic lenses to form the image by controlling the electron beam and converging it on a particular plane with respect to the sample. This mode is similar to the way an optical microscope uses glass lenses to converge light on or through the sample to form an image (Fig. 2).

Following the theoretical elaborations of Louis de Broglie in 1923, proves in 1926 that magnetic or electrostatic fields can be used as lenses for electron beams. The first electron microscope prototype was built in 1931 by the German engineers Ernst Ruska and Max Knoll. This first instrument maximized the objects four hundred times. Two years later, Ruska constructed an electron microscope that exceeded the possible resolution of an optical microscope. Reinhold Rudenberg, the scientific director of Siemens, patented the electron microscope in 1931, stimulated by an illness in the family, to make visible the polio virus. In 1937, Siemens began to finance Ruska and Bodo von Borries to develop an electron microscope. Siemens also used Helmut Ruska's brother to work on applications, especially with biological specifications.

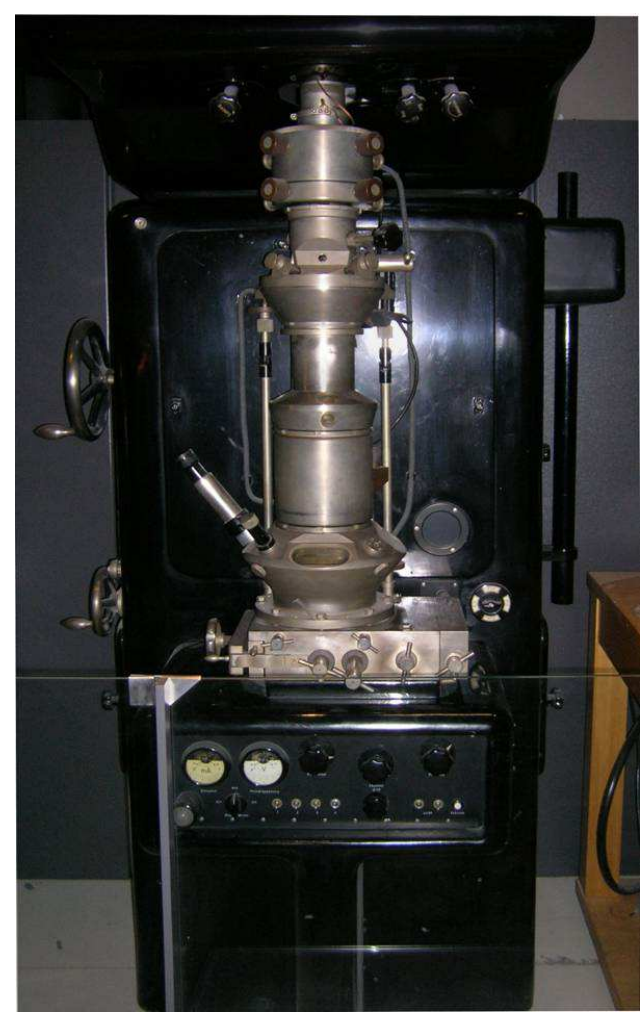

Fig. 2. Electron microscope constructed by Ernst Ruska in 1933; Source: https://en.wikipedia.org/wiki/Ernst_Ruska

During the same decade, Manfred von Ardenne began his research on the scanning electron microscope and his universal electron microscope. Siemens produced the first commercial electronics microscope in 1939 , but in practice the first electron microscope was built at the University of Toronto in 1938 by Eli Franklin Burton and students Cecil Hall, James Hillier and Albert Prebus. The realization of a high resolution electron microscope was only possible after the invention of the stigmator by Hillier, in 1946 in the laboratories of RCA. Although modern electron microscopes can magnify objects up to two million times, they are still on the Ruska prototype. The electron microscope is an essential part of the equipment of many laboratories. Researchers study the reviewers of biological materials, molecule varieties, medical biopsy samples, metals and crystalline structures and characteristics of different surfaces. The electron microscope is also used for inspection, quality assurance and failure analysis of some applications, including, in particular, the manufacture of semiconductor devices.

The original form of electron microscope, the Transmission Electron Microscope (TEM) uses a tungsten as an electron source cathode. The electron beam is accelerated by an anode in general at $100 \mathrm{keV}$ ( 40 to $400 \mathrm{keV}$ ) with respect to the cathode, concentrated by electrostatic and electromagnetic lenses and 
transmitted on the target which is partly transparent to the electrons and Part disperses them. When it emerges from the sample, the electron beam includes information on the structure of the sample which is amplified by the lens system of the microscope lens. The spatial variation of this information (the "image") is seen by projection of the enlarged electronic image on a scintillator, such as zinc sulfide or phosphorus. The image may be recorded photographically by exposing a film or photographic plate directly to the electron beam or a high resolution phosphor plate can be coupled by means of an optical system or optical fiber to the sensor of a Charge-Coupled Device (CCD camera). The image detected by the $\mathrm{CCD}$ can be displayed on a monitor or directed to a computer.

Resolution is limited primarily by spherical aberration, but a new generation of spherical correctors increases the resolution. The spherical aberration correction software for High Resolution MET (HRTEM) enabled the production of images with sufficient resolution to show the carbon atoms in diamonds, separated by only 0.89 ångström ( 89 picometers) and silicon atoms at 0.78 ångström (78 picometers) at magnification of 50 million times. The ability to determine the position of atoms in materials has made HRTEM an important tool for research and development in nanotechnology.

Unlike the MET, where the high-voltage electron beam carries the image of the sample, the electron beam of the Scanning Electron Microscope (SEM) cannot at any moment give an image of the sample. The SEM produces sample images of the sample with an electron beam which, concentrated, is analyzed over a rectangular area of the sample (raster scanning). At each point on the sample the incident electron beam loses energy. This loss of energy is converted into other forms, such as heat, low-energy secondary electron emission, light emission (cathodoluminescence) or Xray emission. Variable intensity of one of these signals in the image in a position corresponding to the position of the beam on the sample when the signal, have been generated. In the image of the right ant, the image was constructed from signals produced by a secondary electron detector, the normal conventional imaging mode of most SEMs. As a general rule, the image resolution of a SEM is about one order of magnitude smaller than that of a MET. However, because the SEM image is based on surface processes rather than transmission, it is capable of delivering images of objects several centimeters with a large depth of field depending on the design and the instrument and it can thus produce images that are a good three-dimensional representation of the structure of the sample.

In the electron microscope by reflection, as in the transmission electron microscope, an electron beam is incident on a surface but instead of using transmission
(MET) or Secondary Electrons (SEM), it is the beam Reflected electrons, dispersed by elasticity, which is detected. This technique is generally associated with the Reflecting High Energy Electron Diffraction (RHEED) and High Energy Reflection of the Loss Spectrum (RHELS). Another variant is Spin-Polarized LowEnergy Electron Microscopy (SPLEEM), which is used to view the microstructure of magnetic domains.

The transmission electron microscopy (SEM) is a type of model whose operating principle combines aspects of the scanning electron microscope and the transmission electron microscope. An electron source focuses an electron beam that passes through the sample. A magnetic lens system allows this beam to scan the surface of the sample to be analyzed.

A Cathode Ray Tube (CRT) is a vacuum tube made of a heated filament, of lens-shaped electrodes which are subjected to a potential difference (voltage) and create an accelerating electric field the electrons. The latter strike the screen, on which is deposited an electroluminescent layer reacting to the impact of electrons by creating a light spot. The trajectory of the electron flow from the cathode to the screen is made possible by the presence of an anode fed to a very high potential (from several thousands to several tens of thousands of volts depending on the type of tube) -this. This component was used for many decades by most televisions, computer screens and oscilloscopes until the advent of LCD screens.

Vacuum techniques had been known since the seventeenth century and, with the mastery of electricity, scientists had the idea of experiencing electricity in tubes containing gas at more or less low pressure. As early as 1858, Germans observed electric discharges in these tubes of Geissler. Electrical discharges in what are now called plasmas actually produce light. It is observed that the magnetic fields produce a deflection on these discharges. It was during such investigations of the conduction of electricity in low-pressure gases that the British physicist and chemist William Crookes discovered that when the pressure was lowered the cathode emitted light rays. They were called cathode rays. It was because he had coated the walls of the tube with a phosphorescent material that Crookes was able to make his observations. Thereafter, the first cathode ray tubes were called the Crookes tube.

The first version of the cathode ray tube is therefore a cold cathode diode with a phosphor layer on the face. The electrons are torn off at the cathode using a very high voltage. After being used for the investigation of physical phenomena, the cathode ray tube will become an instrument for measuring signals that change rapidly over time. The oscillograph will substitute for the rotating mirror for this type of measurements. In 1897 , Ferdinand Braun used for the first time a cathode ray 
tube to study dynamic phenomena, the recording of fast electrical phenomena. The first Braun tubes are filled with low-pressure gas, typically around one-hundredth of a millimeter of mercury. The electron gun consists of a circular pellet which acts as a cathode and a ring electrode acting as an anode. The high voltage applied between the anode and the cathode is produced by an influence machine. In the electric field produced between the cathode and the anode, some positive ions already present in the neutral gas are accelerated towards the cathode, which generates secondary electrons accelerated in the opposite direction. The electrons ionize the molecules of the gas from the residual vacuum and the positive ions, because they are heavy, move away slowly from the beam and the positive space charge thus produced tends to keep the electron beam concentrated throughout of its path to the phosphorescent screen 1 . In the Braun tube, the deflection of electrons in a direction for the signal to be measured and in the direction perpendicular to the reference signal is produced by magnetic coils.

One of the disadvantages of the first oscillographs is then the electron acceleration voltage which must be maintained at a very high level so that the energy of the electron beam at the level of the screen is sufficient. In the early years of the twentieth century, Rankin had the idea of placing a small coaxial coil between the cathode and the anode in order to concentrate the beam. This modification not only increases the intensity of the electron beam but also reduces the magnification of the source image on the screen. The two phenomena go in the direction of an increase in the current density of the beam and the path is thus open to a reduction of the acceleration voltage and therefore of the magnetic deflection fields.

In 1914, A. Dufour had the idea of inserting a photographic plate in the tube instead of the fluorescent screen, which improves the efficiency and consequently the speed of the measurement. This type of apparatus is called a "cathodic oscillograph". This concept causes the development of a number of devices operating between 20 and 60 kilovolts and intended for recording non-periodic fast transient phenomena. The first version using a hot cathode was developed by J. B. Johnson and H. W. Weinhart of Western Electric. This product was marketed in 1922.

In 1907, the Russian inventor Boris Rosing develops a system with a cathode ray tube as an image integrator. In 1911, he demonstrated for the first time the principle of modulation of light by variable speed of the beam. His pupil Vladimir Kosma Zworykin, emigrated to the United States, will produce in 1923 a tube of visualization of the image, the kinescope.

In 1940, in the United States, Peter Goldmark developed a 343-line color television system. This system includes a three-filter disc: red, green and blue, rotating in front of the camera tube.
The cathode rays are high-speed electron flows from a device called an electron gun, consisting of an indirectly heated cathode $(\mathrm{K})$ (a tube with, inside, a filament heated to a voltage of the order of $6.3 \mathrm{~V}$ or more), a gate (G1) called Wehnelt and a series of electrodes charged with the acceleration and focusing of the cathode beam. Heating of the cathode generates the emission of electrons. This emission is focused by the wehnelt. Its potential, lower than that of the cathode $(-35$ to $-90 \mathrm{~V})$, allows to regulate the flow of electrons. The shape of the wehnelt completely surrounding the cathode is intended to prevent the return of positive ions which could lead to its premature destruction6. The flux thus created is then accelerated by an anode (G2) by its very high potential (of the order of $600 \mathrm{~V}$ ) and focused in order to obtain a fine radius by 1 or 2 other anodes (G3, G4). These electrodes act as electrostatic lenses. The beam emitted from the electron gun is then deflected either magnetically by coils (as for a TV tube) or electrostatically by electrodes (in most oscilloscopes). It is this deflection mechanism that performs horizontal and vertical scanning (lines and frames). The beam is subjected to the action of the postaccelerating anode (G5) subjected to a very high voltage (THT) and then arrives on the screen covered with a phosphorescent material, often based on rare earths. When the electrons strike this surface, light is emitted in the form of a concentrated point called "spot" which illuminates the screen at the rate of the sweep conducted by the deflection. The electrons having struck the screen lose their energy and will recombine in an anode, in this case, that of post-acceleration. For a color tube, 3 electron guns (one per primary color) are used and a perforated mask or wires (Trinitron tubes) are added behind the screen in order for each beam to light up the corresponding color (Fig. 3).

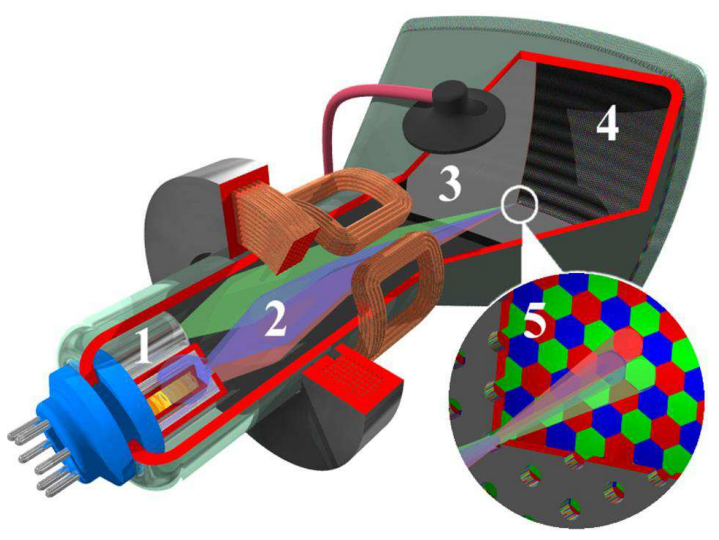

Fig. 3. Color scanning tube 1: electron guns 2: electron beams 3: mask for separating the red, blue and green rays of the displayed image 4: phosphorescent layer with receiving areas for each color 5: close-up on the face Of the screen covered with phosphorus; Source: https://en.wikipedia.org/wiki/Cathode_ray_tube 
Electron beam welding is a process of welding the interaction of an electron beam with the parts to be assembled. High-speed electrons in vacuum have a high chemical energy that will be transferred largely to the work piece at the time of impact, generating heat to cause melting and then welding of the materials. This welding process is essentially automated, taking into account the infrastructure necessary for the generation of the electron beam.

The beam is concentrated on the areas to be assembled. When the power exceeds about $10 \mathrm{~kW}$ per square millimeter, the heat can no longer be dissipated in the substrate. This results in local evaporation of the material and the formation of a cavity in which the solder is formed. The welding takes place in a vacuum enclosure thus avoiding any external contamination or any oxidation. Electron beam welding offers a wide range of power ranging from 10 to $100 \mathrm{~kW}$, giving greater versatility, for example laser welding. The power associated with the possibility of concentrating the beam over a very narrow zone allows a very good penetration (penetration ratio: width of $50: 1$ ). This allows welding on very large thicknesses, for example $200 \mathrm{~mm}$ (steel) or $300 \mathrm{~mm}$ (aluminum). The welding speed also limits the deformation of the parts to be assembled. The conversion rate of electrical energy/heat is also very high, up to $75 \%$ efficiency, compared for example to $10 \%$ for laser methods. The process is fully automated, requiring a higher starting investment, but allowing perfect reproducibility of the weld from one part to another.

The beam generating equipment is very close in principle to a cathode ray tube. A cathode, generally made of a tantalum-tungsten alloy, is heated to its emission temperature. A grid electrode (Wehnelt) is used to adjust the amount of electrons emitted. The emitted electrons are accelerated by an anode whose potential varies between 30 and $200 \mathrm{kV}$ up to plus or minus $2 / 3$ of the speed of the light. The beam is concentrated by a magnetic lens (focusing) before being directed onto the area to be welded. Deflection coils placed near the lens make it possible to vibrate the beam at frequencies between a few $\mathrm{Hz}$ and $10 \mathrm{kHz}$.

In general, the part to be welded is placed on an automated table making it possible to move it under the bundle.

The assembly is placed in a vacuum enclosure, the vacuum around the cathode (10-6 mbar) being greater than the vacuum around the part (10-4 mbar).

The impact of the beam on the part generates an Xray emission which it is necessary to filter through a protective enclosure.

The technology is very well suited for single-pass end welding with thicknesses up to $200 \mathrm{~mm}$ on steel with a $150 \mathrm{kv}$. The joined surfaces are generally milled to allow better contact. A filler metal can be introduced in the form of a strip placed between the pieces or in the form of a wire fed during welding. For pieces of modest thickness, it is possible to obtain very good cosmetic results by using, for example, a second passage of the beam to soften the surface appearance. For larger thicknesses, it is sometimes necessary to incorporate additional geometrical configurations on the original parts, such as a base and a collar which will be eliminated by milling. Most metals can be welded by this method, including metals traditionally difficult to weld without the addition of external materials such as: Refractory metals, low alloy metals, high thermal conductivity metals, copper, "super alloys", tantalum, Titanium, molybdenum, etc. It is not possible to weld galvanized steel, brass, nickel silver with this process. Zinc volatilizes during welding, deposits in thin layer on the walls of the vacuum chamber and can, over time, create short circuits in the electron gun causing the destruction of expensive elements.

The use of electron beam welding finds its usefulness in the welding of large parts where the distortion after welding must be limited to the maximum. The nuclear industry and aerospace are at the origin of the first industrial applications. Thanks to the quality of the results obtained and despite the necessary investment, this technology is also found in the automotive industry, particularly in the welding of transmission parts.

A laser is an apparatus that produces spatially and temporally coherent radiation based on the laser effect. Descending from the maser, the laser was first called optical maser.

A laser source associates an optical amplifier based on the laser effect with an optical cavity, also called a resonator, generally consisting of two mirrors, at least one of which is partially reflective, that is to say a Part of the light exits the cavity and the other part is reinjected towards the inside of the laser cavity. With some long cavities, laser light can be extremely directional. The geometrical characteristics of this set require that the emitted radiation be of a high spectral purity, that is to say temporally coherent. The spectrum of the radiation contains a discrete set of very fine lines, at wavelengths defined by the cavity and the amplifying medium. The fineness of these lines is however limited by the stability of the cavity and by the spontaneous emission within the amplifier (quantum noise). Different techniques make it possible to obtain an emission around a single wavelength.

In the 21 st century, the laser is more generally seen as a possible source for any electromagnetic radiation, of which the light is part. The wavelengths concerned were first microwaves (maser), then they extended to the domains of infrared, visible, ultraviolet and even begin to apply to X-rays (Fig. 4). 


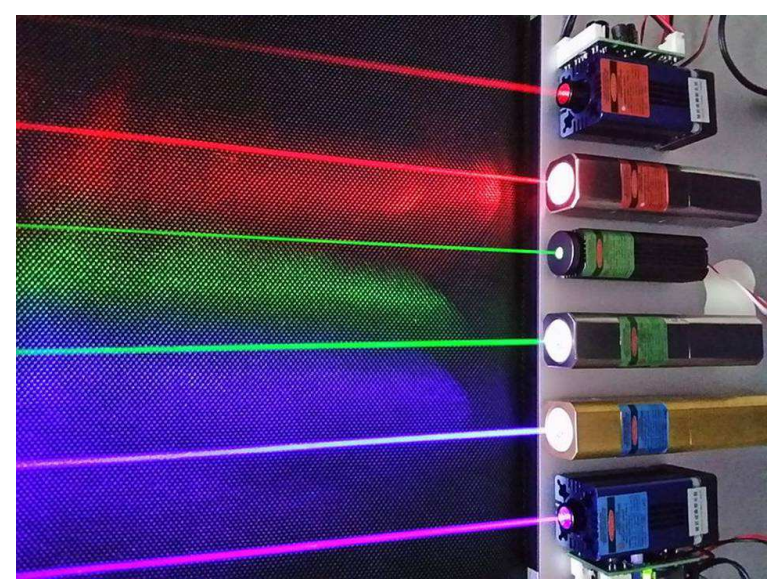

Fig. 4. Red laser (660 and $635 \mathrm{~nm})$, green (532 and $520 \mathrm{~nm})$ and blue (445 and $405 \mathrm{~nm})$; Source: https://en.wikipedia.org/wiki/Laser

The principle of stimulated emission (or induced emission) is described as early as 1916 by Albert Einstein. In 1950, Alfred Kastler (winner of the Nobel Prize for Physics in 1966) proposed an optical pumping process, which he experimentally validated two years later with Brossel and Winter. But it was not until 1953 that the first maser (ammonia gas) was designed by J. P. Gordon, H. J. Zeiger and Ch. H. Townes.

In the following years, many scientists such as N. G. Bassov, Alexander Prokhorov, Arthur Leonard Schawlow and Charles H. Townes help to adapt these theories to visible wavelengths. Towns, Bassov and Prokhorov shared the Nobel Prize for Physics in 1964 for their fundamental work in the field of quantum electronics, which led to the construction of oscillators and amplifiers based on the Maser-Laser principle. In 1960, the American physicist Théodore Maiman obtained for the first time a laser emission by means of a ruby crystal 3. A year later, Ali Javan developed a gas laser (helium and neon) and then in 1966 Peter Sorokin built the first liquid laser.

The lasers find very early industrial outlets. The first application was made in 1965 and consisted of machining a $4.7 \mathrm{~mm}$ diameter hole and $2 \mathrm{~mm}$ deep in diamond with a ruby laser. This was done in $15 \mathrm{~min}$, whereas a typical application took $24 \mathrm{~h}$.

In 1967, Peter Holcroft cut a $2.5 \mathrm{~mm}$ thick stainless steel plate at a speed of $1 \mathrm{~m} / \mathrm{min}$, dioxygen with a 300 W5 $\mathrm{CO}_{2}$ laser and designed the first cutting head.

In the same period in 1963 American researchers such as White and Anderholm show that it is possible to generate a shock wave inside a metal as a result of pulse laser irradiation. The pressures exerted are of the order of $1 \mathrm{GPa}$, or 3 FPs.

Although the processes are demonstrated, their combinations have to be waited on for machines adapted for them to be implanted in an industrial environment. These conditions were fulfilled at the end of the 1970s and the first industrial platforms were established in France as early as the 1980s. From then on, the laser became a tool for industrial production in micro-machining. Its main advantages are high-speed machining of the order of $10 \mathrm{~m} / \mathrm{min}$, without contact, without tool wear.

The laser became a means of reading in 1974, with the introduction of barcode readers. In 1978, laserdiscs were introduced, but optical discs only became commonly used in 1982 with the compact disc. The laser then reads a large volume of data.

Consider a set of two-level atoms. If one sends a field over a set of atoms in the "high" state, the privileged phenomenon will be stimulated emission and the field will be amplified.

To produce an optical amplifier, therefore, it is necessary to find the means of exciting the atoms towards the state of higher energy. More generally, if certain atoms are in the "bottom" ground state, photons can also be absorbed, which decreases the intensity of the field. There will be amplification only if atoms are more likely to be in the "high" state (capable of emitting) than in the "low" state (capable of absorbing): It is necessary to have a "Population inversion".

However, at the thermodynamic equilibrium, the lowest state is always the most populous. At best, populations oscillate between the two levels (Rabi Oscillations).

In order to maintain a population inversion, it is necessary to constantly supply an external energy supply to the atoms, to bring back to the higher state those which are returned to the ground state after the stimulated emission: It is the "pumping". The external energy sources can be of different types, for example an electric generator, or another laser (optical pumping).

The amplifier is therefore a set of atoms or molecules which are made to pass from a fundamental or weakly excited state \{ldisplaystyle\} to a more strongly excited state \{ldisplaystyle $\left.\mathrm{n}^{\prime}\right\}$ \{displaystyle n'\}, By means of an external power source (pumping). These atoms can then de-energize to the \{ \displaystyle n ) n state, by emitting photons with a frequency close to \{\displaystyle $\backslash$ omega _ $\left.\left\{\mathrm{nn}^{\prime}\right\}\right\}$ \{displaystylelomega $\{\mathrm{nn}\}$. Thus, a radiation of this frequency can be amplified by stimulated emission processes.

These lasers use solid media, such as crystals or glasses, as the photon emission medium. The crystal or glass is only the matrix and must be doped by an ion which is the laser medium. The oldest is the ruby laser whose emission comes from the $\mathrm{Cr} 3+$ ion. Other ions are widely used (most of the rare earths: $\mathrm{Nd}, \mathrm{Yb}, \mathrm{Pr}, \mathrm{Er}$, $\mathrm{Tm}$..., titanium and chromium, among others). The emission wavelength of the laser depends essentially on the doping ion, but the matrix also influences. Thus, the neodymium-doped glass does not emit at the same 
wavelength $(1,053 \mathrm{~nm})$ as the neodymium-doped YAG $(1,064 \mathrm{~nm})$. They operate continuously or impulsively (pulses from a few microseconds to a few femtosecondsmillionth of a billionth of a second). They are capable of emitting both in the visible, near infrared and ultraviolet.

The amplifying medium may be a bar in the case of an Nd-YAG laser (thus doped with $\mathrm{Nd}$ and the matrix is YAG: A garnet of aluminum and yttrium), but it can also be in the form of $\mathrm{d} A$ fiber in the case of fiber lasers (thus doped with $\mathrm{Yb}$ and the matrix is made of silica). Today, the most widely used amplifier medium for generating femtosecond pulses is titanium doped sapphire. It has two absorption bands centered at 488 and $560 \mathrm{~nm}$. It has a broad emission spectrum centered at $800 \mathrm{~nm}$.

Beyond a crystal size of acceptable optical quality, these lasers make it possible to obtain powers of the order of $\mathrm{kW}$ in continuous and of GW in pulsed. They are used for both scientific and industrial applications, in particular for welding, marking and cutting of materials.

In liquid lasers, the emission medium is an organic dye (rhodamine 6G for example) in liquid solution enclosed in a glass vial. The emitted radiation can be both continuous and discontinuous depending on the pumping mode.

The transmitted frequencies can be adjusted using a regulating prism, which makes this type of device very precise. The choice of the dye essentially determines the color range of the radius it will emit. The exact color (wavelength) can be adjusted by optical filters.

The photon generating medium is a gas contained in a glass or quartz tube. The beam emitted is particularly narrow and the emission frequency is very limited. The best known examples are helium-neon lasers (red at $632.8 \mathrm{~nm}$ ), used in alignment systems (public works, laboratories) and lasers for shows.

Carbon dioxide lasers are capable of producing very high power (pulse operation) of the order of $106 \mathrm{~W}$. This is the most widely used laser marking in the world. The $\mathrm{CO}_{2}$ laser (infrared at $10.6 \mu \mathrm{m}$ ) can be used, for example, for engraving or cutting materials.

There is also a sub-family of gas lasers: excimer lasers that emit in the ultraviolet. In the majority of cases, they are composed of at least one noble gas and usually a halogen gas.

The term "excimer" comes from the English excited dimer which means an excited molecule composed of two identical atoms (e.g., Xe2). Now, some so-called excimer lasers use exciplexes which are molecules composed of two different atoms (for example, noble gas and halogen: $\mathrm{ArF}, \mathrm{XeCl}$ ). We should therefore call them lasers exciplexes rather than excimer lasers.

The electrical excitation of the mixture produces these exciplex molecules, which exist only in the excited state. After emission of the photon, the exciplexe disappears because its atoms separate, so the photon can not be reabsorbed by the non excited excimer, which allows a good laser yield.

In a laser diode (or semiconductor laser), the pumping takes place by means of an electric current which enriches the generating medium with holes (a hole is a zone of the crystal with a positive charge because it lacks an electron) on one side and additional electrons on the other.

Light is produced at the junction by the recombination of holes and electrons. Often, this type of laser does not have cavity mirrors: The simple fact of cleaving the semiconductor, of high optical index, makes it possible to obtain a coefficient of reflection sufficient to trigger the laser effect.

It is this type of laser that represents the vast majority (in terms of number and turnover) of lasers used in the industry. Indeed, its advantages are numerous: Firstly, it allows a direct coupling between electrical energy and light, hence telecommunications applications (at the input of fiber optic networks). Moreover, this conversion of energy is done with a good yield (of the order of 30 to $40 \%$ ).

These lasers are inexpensive, very compact (the active zone is micrometric, or even less and the whole device has a size of the order of a millimeter). It is now known to produce such lasers in order to obtain light on practically all the visible range, but the lasers delivering red or near infrared remain the most used and the least expensive. There are countless applications: Optical Drives (CD), telecommunications, printers, "pumping" devices for larger lasers (solid-state lasers), pointers, etc. It should be noted that the regulations in force in France prohibit the manufacture of lighting above $1000 \mathrm{~m}$.

They have some disadvantages, however, since the light emitted is generally less directional and less "pure" spectrally than that of other types of lasers (gas in particular); this is not a problem in most applications.

A device which is very close in its operation, but not a laser, is the LED: The pumping device is the same, but the production of light is not stimulated, it is produced by spontaneous de-excitation, so that the light produced does not exhibit the characteristics of coherence characteristic of the laser.

This type of laser is very particular, because its principle is quite different from that explained above. Light is not produced by previously excited atoms, but by synchrotron radiation produced by accelerated electrons. An electron beam from an electron accelerator is fed into an inverter creating a periodic magnetic field (through a permanent magnet assembly).

This inverter is placed between two mirrors, as in the diagram of a conventional laser: The synchrotron 
radiation is amplified and becomes coherent, that is to say it acquires the characteristics of the light produced in the lasers.

It is sufficient to adjust the velocity of the electrons to provide very finely adjusted frequency light over a very wide range, ranging from far infrared (terahertz) to $\mathrm{X}$-rays and the laser power can also be adjusted by the electron flow rate to high levels.

Laser pulses can also be provided with a short and precise interval. All this makes this type of laser very versatile and very useful in research applications. However, it is more expensive to produce because it is necessary to construct a particle accelerator.

This type of laser resembles the solid laser. Here the amplifying medium is an optical fiber doped with rare earth ions. The wavelength obtained depends on the chosen ion (Samarium $0.6 \mu \mathrm{m}$, Ytterbium $1.05 \mu \mathrm{m}$, Erbium $1.55 \mu \mathrm{m}$, Thulium $1.94 \mu \mathrm{m}$, Holmium $2.1 \mu \mathrm{m}$ ). This technology is relatively new (the first date of 1964), but today there are monomode lasers whose power is of the order of about ten kilowatts. These lasers have the advantage of being cheaper, having a smaller footprint and being resistant to vibrations. Furthermore, it is not necessary to cool them below $10 \mathrm{~kW}$.

A photographic sensor is a photosensitive electronic component for converting electromagnetic radiation (UV, visible or IR) into an analog electrical signal. This signal is then amplified, then digitized by an analogdigital converter and finally processed to obtain a digital image. The sensor is therefore the basic component of cameras and digital cameras, the equivalent of film (or film) in silver photography.

The photographic sensor takes advantage of the photoelectric effect which allows the incident photons to tear electrons from each active element (photosite) of a matrix of elementary sensors consisting of photodiodes or photomos. It is much more efficient than the film: Up to $99 \%$ (theoretically) and nearly 50\% (in practice) of the photons received allow one electron to be collected, compared to about $5 \%$ of photons which reveal the photosensitive grain of the film, Hence its initial rise in astrophotography.

Two main families of sensors are available: CCDs and CMOS.

CCDs are still available in compact and high resolution cameras. The most common SLR cameras have abandoned it and mostly use CMOS sensors.

The Charge-Coupled Device (CCD) is the easiest to manufacture. Invented by George E. Smith and Willard Boyle in Bell Laboratories in 1969 (this invention will bring them half the Nobel Prize in physics in 2009), it was quickly adopted for advanced applications (astronomical imaging) and then popularized on cameras and cameras.
A CCD transforms the light photons it receives into electron-hole pairs by the photoelectric effect in the semiconductor substrate and then collects the electrons in the potential well maintained at each photosite. The number of electrons collected is proportional to the amount of light received.

At the end of the exposure, the charges are transferred from photosite to photosite by the set of cyclic potential variations applied to the grids (horizontal conductive strips, isolated by a layer of $\mathrm{SiO}_{2}$ ) to the horizontal register (see animation -against).

Naturally, these sensors are sensitive to the entire spectrum of visible light. Thanks to a Color filter array, for example a Bayer filter, made up of colored cells of the primary colors, each photosite of the sensor sees only one color: Red, green or blue. On each group of four photosites we find one for blue, one for red and two for green; this distribution corresponds to the sensitivity of our vision.

Due to the required precision, the colored pellets of the filter are deposited directly on the sensor with a technology close to the photolithography of the integrated circuits, as is the micro lens array.

It is the software of the photoscope that will recreate the colors, taking into account the spectral response curves for a final result in trichromy; One of the problems is to limit the electronic noise which results in moiré effects on low light areas by judicious compromise during the image processing (interpolation, filtering: See the article Signal processing).

Improvements are regularly made to the CCD sensors in order to improve their sensitivity by increasing the active area:

- Year super-CCD HR (Fujifilm), each photosite has an octagonal surface

- Then (Fujifilm, 2004) the photosites are split into a large "S" element and a smaller " $R$ " element that extends the dynamics to the highlights ( 2 bits) in two successive generations, SR and SR II

- The super-CCD HR (Fujifilm, 2005) benefits from finer electrodes which decrease the depth of the "wells" of the photosites, which thus receive a greater proportion of the light

- The use of Indium Tin Oxide (ITO) electrodes, which are more transparent in blue, improves the spectral response of full-frame CCDs with a Kodak film from 1999

- The Progressive CCD, with a Kodak film from 2005, has finer Load Drainage drains (LOD), again benefiting from the useful surface area

A "Complementary Metal-Oxide-Semiconductor" (CMOS) sensor is composed of photodiodes, like a $\mathrm{CCD}$, where each photosite has its own charge/voltage converter and amplifier (in the case of an APS sensor). 
Their power consumption, much lower than that of CCD sensors, their read speed and the lowest cost of production, are the main reasons for their great use.

In the same way as many CCDs, CMOS color image sensors are associated with a colored filter and a network of lenses, even more necessary given the small relative area of the photodiode, the only sensitive area.

This sensor captures three red, green and blue colors by a single photosite, using three layers of silicon coated with photosites and sandwiched and each filtered by a blue, green or red filter; each of the photoreceptor layers is precisely spaced relative to the blue, green and red wavelengths of the visible light. To simplify, we can say that by receiving an incident ray, the silicon surface layer stops the blue, the middle layer stops the green and finally the red is stopped by the lower layer, as shown in the figure below against.

The X3 sensor was developed by the American company Foveon, bought in 2008 by Sigma, which has since enjoyed an exclusive exploitation right.

Unlike a photosite CCD sensor that captures only a primary color (red, green or blue), an X3 sensor photosite collects an RGB component. This requires much less computing electronics, since the color is directly obtained on the photosite and more after electronic processing of the colors of four photosites. This is an advantage not only in terms of manufacturing costs but also in terms of quality. Indeed, the absence of calculations and interpolations makes it possible to hope for "cleaner" images and would also allow a faster rate of shooting in burst mode. The higher the number of pixels, the better the definition of a picture, which can be useful when enlarging an image. However, the number of photosensitive cells per square millimeter of the sensor also has an influence on the quality of the images: There is therefore no exclusive link between number of pixels and output image quality and it is generally useless to Compare two sensors as well as by their number of pixels: The quality of an image also depends on the quality and intensity of the light that the sensor can receive on each of its photosensitive cells.

A sensor with a small area but with a high density of pixels per square millimeter can be interesting in terms of mass production and can lower the price without necessarily diminishing the quality of the photo. See the technical limitations described below.

A particle accelerator is an instrument that uses electric or magnetic fields to bring electrically charged particles at high speeds. In other words, it communicates energy to the particles. There are two main categories: Linear accelerators and circular accelerators.
In 2004, there were over 15,000 accelerators worldwide.

Only a hundred are very large national or supranational facilities (CERN). Industrial-type electrostatic machines account for more than $80 \%$ of the world's industrial electron accelerators. Many small linear accelerators are used in medicine (antitumor radiotherapy).

\section{Conclusion}

Using the original method proposed by the authors of this paper, the moving electron beam can be determined with great accuracy depending on its speed.

It can be seen from the results presented in Table 1 that the electron has no constant radius.

The electronic phase depends primarily on the speed of movement and, secondly, on the resting table.

The proposed relationship 1 can accurately determine the R-rays of the moving electron, depending on the speed at which it moves and the rest mass $\mathrm{m} 0$.

The mechanical moment of inertia of an electron around one of its axes can be determined by relationship 2.

Expression 4 shows the total kinetic energy of a moving electron as the sum of the two components (translational and rotational). Using the theory of Louis de Broglie, which shows impulse preservation, the wavelength associated with the wavelength can be calculated with relation 7 . The frequency of the wave (associated with the moving electron) can be determined using the relationship 8 .

The electronic kinetic energy of the motion can also be calculated by the expression 11, lowering the total resting energy of the electron from the energy of the electron in full motion.

Determining the dimensions of a moving electron with a very high accuracy becomes reality using the original methods and relationships presented in this study.

This is a vital process that is being sought and wanted by electronics, physics, chemistry, material science and nanotechnology specialists.

Applications of the present work in multiple domains will be accessed through the original exposed method.

\section{Acknowledgement}

This text was acknowledged and appreciated by Dr. Veturia CHIROIU Honorific member of Technical Sciences Academy of Romania (ASTR) PhD supervisor in Mechanical Engineering.

\section{Funding Information}

Petrescu F.I., 2012, Cold nuclear fusion. Plasma Physics and Fusion Technology (S70), INIS 44(16) 


\section{Author's Contributions}

All the authors contributed equally to prepare, develop and carry out this manuscript.

\section{Ethics}

This article is original. Authors declare that are not ethical issues and no conflict of interest that may arise after the publication of this manuscript.

\section{Nomenclature}

$\varepsilon_{0} \quad=>$ the permissive constant (the permittivity):

$\varepsilon_{0} \quad=8.85418 \mathrm{E}-12\left[\mathrm{C}^{2} / \mathrm{Nm}^{2}\right]$

$h \quad \Rightarrow$ the Planck constant:

$h \quad=6.626 \mathrm{E}-34$ [Js]

$q \quad=>$ electrical elementary load:

$q_{e} \quad=-1.6021 \mathrm{E}-19[\mathrm{C}]$

$q_{p} \quad=1.6021 \mathrm{E}-19[\mathrm{C}]$

$c=$ the light speed in vacuum:

c $\quad=2.997925[\mathrm{~m} / \mathrm{s}]$

$m_{0}[\mathrm{~kg}] \quad=>$ the rest mass of one particle

$m_{0 \text { electron }}=9.11 \mathrm{E}-31[\mathrm{~kg}]$

\section{References}

Albert Einstein, From Wikipedia, the free encyclopedia. Retrieved from: https://en.wikipedia.org/wiki/Albert_Einstein

Arthur Compton, From Wikipedia, the free encyclopedia. Retrieved from: https://en.wikipedia.org/wiki/Arthur_Compton

Aversa, R., F.I.T. Petrescu, R.V.V. Petrescu and A. Apicella, 2016a. Biomimetic finite element analysis bone modeling for customized hybrid biological prostheses development. Am. J. Applied Sci., 13: 1060-1067.

DOI: 10.3844/ajassp.2016.1060.1067

Aversa, R., R.V.V. Petrescu, F.I.T. Petrescu and A. Apicella, 2016b. Smart-factory: Optimization and process control of composite centrifuged pipes. Am. J. Applied Sci., 13: 1330-1341.

DOI: 10.3844 /ajassp.2016.1330.1341

Aversa, R., F. Tamburrino, R.V.V. Petrescu, F.I.T. Petrescu and M. Artur et al., 2016c. Biomechanically inspired shape memory effect machines driven by muscle like Acting NiTi alloys. Am. J. Applied Sci., 13: 1264.1271.DOI: 10.3844/ajassp.2016.1264.1271

Aversa, R., F.I.T. Petrescu, R.V.V. Petrescu and A. Apicella, 2016d. Biofidel FEA modeling of customized hybrid biological hip joint design Part II: Flexible stem trabecular prostheses. Am. J. Biochem. Biotechnol., 12: 277-285.

DOI: 10.3844/ajbbsp.2016.277.285
Aversa, R., F.I.T. Petrescu, R.V.V. Petrescu and A. Apicella, 2016e. Biofidel FEA modeling of customized hybrid biological hip joint prostheses, Part I: Biomechanical behavior of implanted femur. Am. J. Biochem. Biotechnol., 12: 270.276.

DOI: 10.3844/ajbbsp.2016.270.276

Bose-Einstein condensate, From Wikipedia, the free encyclopedia. Retrieved from: https://en.wikipedia.org/wiki/Bose $\%$ E2\%80\%93Ein stein_condensate

Cherenkov radiation, From Wikipedia, the free encyclopedia. Retrieved from: https://en.wikipedia.org/wiki/Cherenkov_radiation

Ernest Rutherford, From Wikipedia, the free encyclopedia. Retrieved from: https://en.wikipedia.org/wiki/Ernest_Rutherford

Fujifilm, 2004. Retrieved from: http://www.fujifilm.com/news/2004/

Fujifilm, $2005 . \quad$ Retrieved from: http://www.fujifilm.com/news/2005/

Halliday, D. and R. Robert, 1966. Physics, Part II. 1st Edn., John Wiley and Sons, Inc., New York.

Louis de Broglie, Wikipedia. Retrieved from: https://en.wikipedia.org/wiki/Louis_de_Broglie

Lorentz transformation, Wikipedia. Retrieved from: https://en.wikipedia.org/wiki/Lorentz_transformation

Max Planck constant, From Wikipedia, the free encyclopedia. Retrieved from: https://en.wikipedia.org/wiki/Planck_constant

Mirsayar, M.M., V.A. Joneidi, R.V.V. Petrescu, F.I.T. Petrescu and F. Berto, 2017. Extended MTSN criterion for fracture analysis of soda lime glass. Eng. Fracture Mechan., 178: 50-59.

DOI: 10.1016/j.engfracmech.2017.04.018

Niels Bohr, From Wikipedia, the free encyclopedia. Retrieved from: https://en.wikipedia.org/wiki/Niels_Bohr

Paul Dirac, From Wikipedia, the free encyclopedia. Retrieved from: https://en.wikipedia.org/wiki/Paul_Dirac

Petrescu, F.I. and J.K. Calautit, 2016a. About nano fusion and dynamic fusion. Am. J. Applied Sci., 13: 261-266 DOI: 10.3844/ajassp.2016.261.266

Petrescu, F.I. and J.K. Calautit, 2016b. About the light dimensions. Am. J. Applied Sci., 13: 321-325. DOI: 10.3844/ajassp.2016.321.325

Petrescu, R.V., R. Aversa, A. Apicella, S. Li and G. Chen et al., 2016a. Something about electron dimension. Am. J. Applied Sci., 13: 1272-1276.

Petrescu, F.I., A. Apicella, R.V. Petrescu, S. Kozaitis and R. Bucinell et al., 2016b. Environmental protection through nuclear energy. Am. J. Applied Sci., 13: 941-946.

DOI: 10.3844/ajassp.2016.941.946 
Relly Victoria Virgil Petrescu et al. / American Journal of Engineering and Applied Sciences 2017, 10 (2): 584.602

DOI: 10.3844/ajeassp.2017.584.602

Petrescu, F.I., 2012. Teoria Mecanismelor Color: Curs si Aplicatii. 1st Edn., CreateSpace Publisher, pp: 284.

Richard Laming, From Wikipedia, the free encyclopedia. Retrieved

from: https://en.wikipedia.org/wiki/Richard_Laming

Thomson, J.J., 1897. From Wikipedia, the free encyclopedia.

Retrieved

https://en.wikipedia.org/wiki/J._J._Thomson from:
Wolfgang Pauli, From Wikipedia, the free encyclopedia. Retrieved from: https://en.wikipedia.org/wiki/Wolfgang_Pauli 